Egypt. Acad. J. biolog. Sci., 2 (2): 43- 56 (2010)

Email: egyptianacademic@yahoo.com

Received: 29/6/2010
F. Toxicology \&Pest control

ISSN: 2090 - 0791

www.eajbs.eg.net

\title{
Effect of Chitin synthesis inhibitors (flufenoxuron) on some biological and biochemical aspects of the cotton leaf worm Spodoptera littoralis Bosid (Lepidoptera: Noctuidae)
}

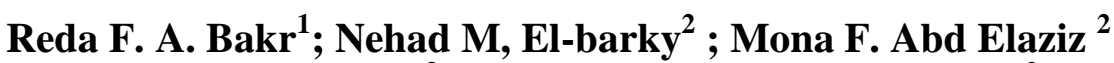 \\ Mohamed H. Awad ${ }^{3}$ and Hisham M. E. Abd El-Halim².
}

1- Entomology Department - Faculty of Science- Ain Shams University

2- Entomology Department - Faculty of Science- Benha University

3- Zoology Department - Faculty of Science- Benha University

\begin{abstract}
The present study aimed to evaluate the biological effect of insect growth regulator flufenoxuron (Cascade) as a chitin synthesis inhibitor against $2^{\text {nd }}$ and $4^{\text {th }}$ larval instars of Spodoptera littoralis, to determine its toxicity. Effect of sublethal doses $\mathrm{LC}_{25}, \mathrm{LC}_{50}$ and $\mathrm{LC}_{90}$ were used to investigate the enzymatic activities. The tested IGR significantly increased the larval and pupal durations, on the other hand decrease the percentages of pupation, adult emergency, fecundity and fertility of the eggs produced by the adult progeny. The tested compound significantly induced larval mortalities, which were dose dependant.

Treatments of the $2^{\text {nd }}$ and $4^{\text {th }}$ larval instars with the tested IGR induced some morphogenic abnormalities in larval, larval-pupal and pupal stages, as well as pupal-adult intermediate. Some emerged adults have various degrees of malformations. All the treated larvae as $2^{\text {nd }}$ instar showed a high sensitivity to the tested IGRs more than $4^{\text {th }}$ instars. The treated larvae in both $2^{\text {nd }}$ and $4^{\text {th }}$ larval instars with the sublethal doses $\mathrm{LC}_{25}, \mathrm{LC}_{50}$ and $\mathrm{LC}_{90}$ showed a significant decrease in enzyme activities of acid phosphatase and the non- specific esterases, $\alpha, \beta$ esterases at different times intervals post treatments.
\end{abstract}

Keywords: IGR, flufenoxuron- biological and biochemical aspects- Spodoptera littoralis,

\section{INTRODUCTION}

The Egyptian cotton leaf worm, Spodoptera littoralis Bosid (Lepidoptera: Noctuidae) is a polyphagous foliage feeding insect. It considered as one of the most serious pests of many different Egyptian crops (Magd El- Din \& El-Gengaihi, 2000). It is an important pest of cotton in Africa, Middle East and Southern Europe (Hosny et al., 1986).

The recent control intensive research is concerned mainly with avoiding the serious problems resulted from using harmful insecticides that cause harmful residues in the food chain and pollution of the surrounding natural enemies and pest resistance. Therefore, now it has become necessary to search for alternative means of pest control which can minimize the use of these synthetic chemicals (Abo-Arab and Salem, 2005).

The necessity to find environmentally safe insecticides as well as materials to combat species resistant to conventional pesticides has spurred increased interest in alternative insecticides such as use of plant extraction and insect growth regulators (IGRs). IGRs are considered to have little human toxicity because humans do not make chitin and do not make or use the hormones insects use in moulting (Schmutterer, 1985).

The use of IGRs compounds in insect control is known as insect 
developmental inhibition, which inhibits or prevents normal metamorphosis of immature stages to the adult stage. These compounds have been tested successfully against several insect species (Pineda et al., 2007; Elbarky et al., 2009 and Wang \&Tian 2009)

Chitin synthesis inhibitors (CSIs) interfere with chitin biosynthesis in insects (Gijswijt et al., 1979) and thus prevent moulting or produce an imperfect cuticle (Hammock and Quistad, 1981). These compounds are effective suppressors of development for the entire life cycle of insects (Verloop and Ferrell, 1977). However, these compounds, also, affect the hormonal balance resulting in physiological disturbances (DeLoach et al., 1981).

The present study was undertaken to investigate the effect of flufenoxuron for controlling $S$. littoralis larvae and study the susceptibility of $2^{\text {nd }}$ and $4^{\text {th }}$ larval instars to different concentrations. This can be attained by determining it's possible larvicidal effect, it's possible latent effect on certain biological aspects and the effect of $\mathrm{LC}_{25}, \mathrm{LC}_{50}$ and $\mathrm{LC}_{90}$ of the tested compound on some enzymatic activities $(\alpha-, \beta$ esterases and acid phosphatases).

\section{MATERIALS AND METHODS Test insect}

The culture of the cotton leaf worm, $S$. littoralis Bosid was initiated from freshly collected egg masses supplied from the division of cotton leaf worm, of Plant Protection Research Institute, Dokki, Egypt. All rearing steps of the colony and experiments were kept under laboratory conditions of $27 \pm 2 \mathrm{C}^{\circ}$ and R.H. $70 \pm 5 \%$.

\section{Tested Compound}

Chitin synthesis inhibitors, Benzoylphenylurea derivatives,
Cascade 10\% (flufenoxuron) was used in this study.

\section{Biological studies:}

Newly moulted $2^{\text {nd }}$ and $4^{\text {th }}$ larval instars were segregated from the stock colony in clean glass Petri dishes and starved for $24 \mathrm{hrs}$ (Nasr, 1999). Five concentrations of IGR were used. The concentrations were prepared by dissolving the tested IGR in distilled water to get the appropriate concentrations. Pieces of castor been leaves were treated by the leaf-dipping technique in the different concentrations of tested compound and left in the air for $1 \mathrm{~h}$ to insure that it is completely dry, and then introduced to larvae for feeding. Eighty of starved larvae, distributed in four replicates (20 larvae/replicate) were used for each concentration and allowed to feed for $24 \mathrm{hrs}$ on treated castor bean leaves. Unconsumed food, dead larvae and faces were removed daily before introducing fresh leaves. The same technique described above was used except that the control larvae were allowed to feed on castor bean leaves that dipped only in distilled water.

Daily inspections were carried out until adult emergence occurred and the number of individuals that managed to develop was recorded. Larval mortality\%, larval duration, pupation $\%$, pupal duration and pupal malformation were recorded.

Adult emergence \%, total inhibition of adult emergence $\%$, fertility $\%$, fecundity, sterility $\%$ and in addition malformations was recorded. Adult fecundity was determined by placing one female and one male together in a glass jar of 75 c.c capacity provided with a piece of cotton soaked in 10\% sugar solution (as a source of food for moths) and was internally covered with soft sheet of paper for oviposition. The jars were inspected daily for counting the 
number of laid eggs. To determine the fertility, two or three patches having not less than 100 eggs were collected during the first 3 days of oviposition and incubated under the laboratory conditions until hatching and the percentage of hatchability was recorded.

\section{Toxicological studies:}

Newly moulted $2^{\text {nd }}$ and $4^{\text {th }}$ larval instars were treated with different concentrations as described later in biological studies technique. Mortality percentages of the treated and control larvae were recorded at 24 hrs post-treatment.

\section{Estimation of enzymatic activities:}

Some biochemical traits of haemolymph such as acid phosphatase, $\alpha-\& \beta$ - esterases were measured at different time intervals 6-12-24-48 hrs post treatment with $\mathrm{LC}_{25}, \mathrm{LC}_{50}$ and

\section{RESULTS}

\section{Effect of flufenoxuron on some biological aspects:}

Effects of flufenoxuron on some biological aspects of $S$. littoralis treated as $2^{\text {nd }}$ larval instar were recorded in Tables (1\&2). Data obtained in Table (1) showed that the corrected percentages of larval mortality had a positive relationship with the different concentrations of flufenoxuron. The response was dosedependent (i.e. the higher concentration affected more larvae). On the other hand
$\mathrm{LC}_{90}$ ppm concentrations. Acid phosphatase was determined according to the method described by Powell and Smith (1954). Alpha esterases $(\alpha-$ esterases) and beta esterases ( $\beta$ esterases) were determined according to the methods of Van Asperen (1962) using $\alpha$-naphthyl acetate and $\beta$ naphthyl acetate as substrates, respectively.

\section{Statistical analysis:}

By using Origen lab program version7.5 the data were expressed as means \pm standard errors. The statistical significance of differences between individuals means were determined by using one way ANOVA test. Levels of significance of each experiment was stated to be significant at $(P=0.05)$, high significant at $(\mathrm{P}=0.01)$ and very high significant at $(\mathrm{P}=0.001)$.

the data obtained in the same table, indicated that there was an inverse relationship between the different concentrations of flufenoxuron and pupation percentages. While the percentages of pupal mortality were increased with the increase in concentrations. Also the percentages of the adult emergence were decreased with the increasing in concentrations as compared with control. Moreover higher concentrations induce more inhibition of adult emergence.

Table (1): The effect of flufenoxuron on biological aspects of cotton leafworm by feeding newly $2^{\text {nd }}$ instar larvae on treated Castor Leave for $24 \mathrm{hrs}$.

\begin{tabular}{|c|c|c|c|c|c|c|c|}
\hline $\begin{array}{l}\text { Conc. } \\
\text { (ppm) }\end{array}$ & $\begin{array}{c}\text { Larval } \\
\text { mortality } \\
\% \\
\pm \text { S.E }\end{array}$ & $\begin{array}{c}\begin{array}{c}\text { Larval } \\
\text { duration } \\
\text { (days) }\end{array} \\
\pm \text { S.E }\end{array}$ & $\begin{array}{c}\text { Pupation } \\
\% \\
\pm \text { S.E }\end{array}$ & $\begin{array}{c}\text { Pupal } \\
\text { mortality } \\
\% \\
\pm \text { S.E }\end{array}$ & $\begin{array}{c}\begin{array}{c}\text { Pupal } \\
\text { duration } \\
\text { (days) }\end{array} \\
\pm \text { S.E. }\end{array}$ & $\begin{array}{c}\text { Emerged } \\
\text { moths } \\
\% \\
\pm \text { S.E }\end{array}$ & $\begin{array}{c}\text { total } \\
\text { inhibition } \\
\text { of adult } \\
\text { emergence \% }\end{array}$ \\
\hline 0.0 & $\begin{array}{l}--- \\
\pm 0.0\end{array}$ & $\begin{array}{c}10 \\
\pm 0.41\end{array}$ & $\begin{array}{c}100 \\
\pm 0.0\end{array}$ & $\begin{array}{l}--- \\
\pm 0.0\end{array}$ & $\begin{array}{c}7 \\
\pm 0.41\end{array}$ & $\begin{array}{c}100 \\
\pm 0.0\end{array}$ & $\begin{array}{l}---- \\
\pm 0.0\end{array}$ \\
\hline 0.1 & $\begin{array}{r}30 * \\
\pm 0.41\end{array}$ & $\begin{array}{c}11 \\
\pm 0.41\end{array}$ & $\begin{array}{r}* * * * \\
70 \\
\pm 0.41\end{array}$ & $\begin{array}{r}* 6.25 \\
\pm 0.25\end{array}$ & $\begin{array}{r}10 * * * \\
\pm 0.41\end{array}$ & $\begin{array}{l}\quad 63.75 \\
\pm 0.25\end{array}$ & $\begin{array}{l}36.25 \\
\pm 1.25\end{array}$ \\
\hline 0.5 & $\begin{array}{r}60 * \\
\pm 0.71\end{array}$ & $\begin{array}{l}11.75 \\
\pm 0.25\end{array}$ & $\begin{array}{r}* * * * \\
40 \\
\pm 0.71\end{array}$ & $\begin{array}{r}\text { F**** }^{* *} 10 \\
\pm 0.41\end{array}$ & $\begin{array}{r}12 * * \\
12 \\
\pm 0.41\end{array}$ & $\begin{array}{r}3 * * * 30 \\
\pm 0.41\end{array}$ & $\begin{array}{r}70 * * \\
\pm 2.04\end{array}$ \\
\hline 1.0 & $\begin{array}{r}80 \\
\pm 0.41\end{array}$ & $\begin{array}{c}12.5 \\
\pm 0.29\end{array}$ & $\begin{array}{r}20 \\
\pm 0.41\end{array}$ & $\begin{array}{l}11.25 \\
\pm 0.25\end{array}$ & $\begin{array}{r}12 \\
\pm 0.41\end{array}$ & $\begin{array}{l}\quad 8.75 \\
\pm 0.25\end{array}$ & $\begin{array}{l}91.25 \\
\pm 1.25\end{array}$ \\
\hline 1.5 & $\begin{array}{r}95 \\
\pm 0.0\end{array}$ & $\begin{array}{c}13 \\
\pm 0.91\end{array}$ & $\begin{array}{r}5 \\
\pm 0.0\end{array}$ & $\begin{array}{c}1.25 \\
\pm 0.25\end{array}$ & $\begin{array}{r}14 \\
\pm 0.56\end{array}$ & $\begin{array}{l}3.75 \\
\pm 0.25\end{array}$ & $\begin{array}{l}96.25 \\
\pm 1.25\end{array}$ \\
\hline 2.0 & $\begin{array}{c}100 \\
\pm 0.0\end{array}$ & $\begin{array}{c}8 \\
\pm 0.71\end{array}$ & $\begin{array}{r}0 \\
\pm 0.0\end{array}$ & $\begin{array}{c}0 \\
\pm 0.0\end{array}$ & $\begin{array}{r}0 \\
\pm 0.0\end{array}$ & $\begin{array}{r}0 \\
\pm 0.0\end{array}$ & $\begin{array}{r}100 \\
\pm 0.0\end{array}$ \\
\hline
\end{tabular}


The larval and pupal durations were increased with the increasing of concentrations as compared with control, (i.e. the higher concentration induce more prolongation in both larval and pupal durations).

The fecundity and fertility were decreased as a result of treatment with flufenoxuron as indicated in Table (2). This decrease was negatively correlated with the concentration. On the other hand, the oviposition deterrent index (O.D.I) and percentages of sterility were positively correlated with the concentrations) for instance; (O.D.I) was $1.02,2.53,10.19,12.22$ and 0.0 $\%$ at the concentrations of $0.1,0.5$, $1.0,1.5$ and $2.0 \mathrm{ppm}$, respectively. Also, the percentage of sterility was $5.22,12.8,28,36$ and $0.0 \%$ at the previous concentrations.

Table (2): Effect of flufenoxuron on fecundity, fertility and sterility against adults of cotton leafworm emerged from $2^{\text {nd }}$ larval instar feeding on treated castor leaves for $24 \mathrm{hrs}$

\begin{tabular}{|c|c|c|c|c|}
\hline $\begin{array}{c}\text { Conc. } \\
(\mathbf{p p m})\end{array}$ & $\begin{array}{c}\text { No. of eggs/female } \\
\text { (fecundity) } \pm \text { S.E }\end{array}$ & + O.D.I \% \pm S.E & $\begin{array}{c}\text { Egg hatching } \\
\text { (fertility) \% } \pm \text { S.E }\end{array}$ & Sterility \% \pm S.E \\
\hline $\mathbf{0 . 0}$ & $1250 \pm 17.68$ & $0 \pm 0.0$ & $100 \pm 0.0$ & $0 \pm 0.0$ \\
\hline $\mathbf{0 . 1}$ & $1241 \pm 7.97$ & $1.02 \pm 0.21$ & $95.5 \pm 2.05$ & $*^{*} 12.8 \pm 0.9$ \\
\hline $\mathbf{0 . 5}$ & $1188 \pm 8.16$ & $2.53 \pm 1.01$ & $91.6 \pm 1$ & ${ }^{* * *} 28 \pm 3.45$ \\
\hline $\mathbf{1 . 0}$ & ${ }^{* * * *} 1019 \pm 22.63$ & ${ }^{* * *} 10.19 \pm 1.47$ & ${ }^{* * *} 88.35 \pm 3.04$ & ${ }^{* * *} 36 \pm 3.8$ \\
\hline $\mathbf{1 . 5}$ & ${ }^{* * *} 980 \pm 45.28$ & $12.22 \pm 3.01$ & ${ }^{* * * *} 0 \pm 0.0$ & $0 \pm 0.0$ \\
\hline $\mathbf{2 . 0}$ & ${ }^{* * *} 0 \pm 0.0$ & $0 \pm 0.0$ & \\
\hline
\end{tabular}

*Significant at $\mathrm{P}=0.05 \quad * *$ High significant at $\mathrm{P}=0.01 * * *$ Very high significant at $\mathrm{P}=0.001$

Data in Table $(3 \& 4)$ showed the effects of flufenoxuron on some biological aspects of $S$. littoralis treated as $4^{\text {th }}$ larval instar. Data in Table (3) declared that there was a highly significant effect on the larval mortality that given in corrected percentages.

Table 3: The effect of flufenoxuron on some biological aspects of the cotton leafworm by feeding newly $4^{\text {th }}$ instar larvae on treated castor leaves for $24 \mathrm{hrs}$.

\begin{tabular}{|c|c|c|c|c|c|c|c|}
\hline $\begin{array}{l}\text { Conc. } \\
\text { (ppm) }\end{array}$ & $\begin{array}{c}\text { Larval } \\
\text { mortality } \\
\% \\
\pm \text { S.E }\end{array}$ & $\begin{array}{c}\begin{array}{c}\text { Larval } \\
\text { duration } \\
\text { (days) }\end{array} \\
\pm \text { S.E }\end{array}$ & $\begin{array}{c}\text { Pupation } \\
\% \\
\pm \text { S.E }\end{array}$ & $\begin{array}{c}\text { Pupal } \\
\text { mortality } \\
\% \\
\pm \text { S.E }\end{array}$ & $\begin{array}{c}\begin{array}{c}\text { Pupal } \\
\text { duration } \\
\text { (days) }\end{array} \\
\pm \text { S.E. }\end{array}$ & $\begin{array}{c}\text { Emerged } \\
\text { moths } \\
\% \\
\pm \text { S.E }\end{array}$ & $\begin{array}{c}\text { Total } \\
\text { inhibition } \\
\text { of adult } \\
\text { emergence \% }\end{array}$ \\
\hline 0.0 & \pm 0.0 & $\begin{array}{c}6 \\
\pm 0.41\end{array}$ & $\begin{array}{c}100 \\
\pm 0.0\end{array}$ & $\begin{array}{l}---- \\
\pm 0.0\end{array}$ & $\begin{array}{c}8 \\
\pm 0.58\end{array}$ & $\begin{array}{c}100 \\
\pm 0.0\end{array}$ & $\begin{array}{l}---- \\
\pm 0.0\end{array}$ \\
\hline 1 & $\begin{array}{r}* * * 10 \\
\pm 0.41\end{array}$ & $\begin{array}{c}6.5 \\
\pm 0.29\end{array}$ & $\begin{array}{l}{ }^{* *} 90 \\
\pm 0.41\end{array}$ & $\begin{array}{c}2.5 \\
\pm 0.29\end{array}$ & $\begin{array}{c}8 \\
\pm 0.41\end{array}$ & ${ }^{* * *} 87.5 \pm 0.29$ & $\begin{array}{r}* * * \\
12.5 \\
\pm 1.44\end{array}$ \\
\hline 3 & $\begin{array}{l}* * * * 32.5 \\
\pm 0.29\end{array}$ & $\begin{array}{c}* 8 \\
\pm 0.41\end{array}$ & $\begin{array}{l}* * * * 67.5 \\
\pm 0.29\end{array}$ & $\begin{array}{l}* * 12.5 \\
\pm 0.29\end{array}$ & $\begin{array}{c}10 \\
\pm 0.41\end{array}$ & $\begin{array}{l}* * * * 55 \\
\pm 0.0\end{array}$ & $\begin{array}{l}* * * 45 \\
\pm 0.0\end{array}$ \\
\hline 5 & $\begin{array}{l}* * * 55 \\
\pm 0.41\end{array}$ & $\begin{array}{l}{ }^{* * *} 10 \\
\pm 0.41\end{array}$ & $\begin{array}{l}* * * 45 \\
\pm 0.41\end{array}$ & $\begin{array}{l}\text { ****1 } 16.25 \\
\pm 0.62\end{array}$ & $\begin{array}{c}* 11 \\
\pm 0.41\end{array}$ & $\begin{array}{c}* * * 28.75 \\
\pm 0.25\end{array}$ & $* * * * 71.25 \pm 1.25$ \\
\hline 7 & $\begin{array}{l}* * * * 80 \\
\pm 0.41\end{array}$ & $\begin{array}{l}* * * 12 \\
\pm 0.41\end{array}$ & $\begin{array}{l}{ }^{* * *} 20 \\
\pm 0.41\end{array}$ & $\begin{array}{c}{ }^{*} 10 \\
\pm 0.41\end{array}$ & $\begin{array}{l}* * * * 12 \\
\pm 0.41\end{array}$ & $\begin{array}{c}* * * * 10 \\
\pm 0.0\end{array}$ & $\begin{array}{l}{ }^{* * * *} 90 \\
\pm 0.0\end{array}$ \\
\hline 9 & $\begin{array}{c}* * * * \\
\quad 100 \\
\pm 0.0\end{array}$ & $\begin{array}{c}* 4 \\
\pm 0.41\end{array}$ & $\begin{array}{c}* * * *- \\
\pm 0.0\end{array}$ & \pm 0.0 & $\begin{array}{c}* * * * \\
\pm 0.0\end{array}$ & $\begin{array}{c}* * * \\
\pm 0.0\end{array}$ & $\begin{array}{l}* * * \\
\quad 100 \\
\pm 0.0\end{array}$ \\
\hline
\end{tabular}

$*$ Significant at $\mathrm{P}=0.05 \quad * *$ High significant at $\mathrm{P}=0.01 * * *$ Very high significant at $\mathrm{P}=0.001$

+ Oviposition deterrenet index 
Pupation percentage was greatly reduced as compared with control, while the percentages of pupal mortality were increased with the increase in concentrations. The reduction in the adult emergence percentages was increased with the increasing in the concentrations, total inhibitions of adult emergence were $12.5,45,71.25,90$ and $100 \%$ at the Data in Table (4) showed the effect of flufenoxuron on the fecundity, (O.D.I), fertility and sterility. conc. of $1,3,5,7$ and 9 ppm, respectively, as compared with $0.0 \%$ in the control. The response was dosedependent. It is remarkable that the larval and pupal durations were increased with the increasing of concentrations as compared with control, (i.e. higher concentration induce more prolongation in both larval and pupal durations).

The fecundity and fertility were decreased. This decrease was negatively correlated with the concentration.

Table (4): Effect of Cascade on fecundity, fertility and sterility against adults of cotton leafworm emerged from $4^{\text {th }}$ larval instar feeding on treated castor leaves for $24 \mathrm{hrs}$.

\begin{tabular}{|c|c|c|c|c|}
\hline $\begin{array}{c}\text { Conc. } \\
(\mathrm{ppm})\end{array}$ & $\begin{array}{c}\text { No. of eggs/female } \\
\text { (fecundity) } \\
\pm \text { S.E }\end{array}$ & $\begin{array}{c}{ }^{+} \text {O.D.I } \% \\
\pm \text { S.E }\end{array}$ & $\begin{array}{c}\text { Egg hatching } \\
\text { (fertility) } \\
\pm \text { S.E }\end{array}$ & $\begin{array}{c}\text { Sterility } \\
\% \\
\pm \text { S.E }\end{array}$ \\
\hline 0.0 & $1430 \pm 24.83$ & $0 \pm 0.0$ & $100 \pm 0.0$ & $0 \pm 0.0$ \\
\hline 1 & $1385 \pm 30.48$ & $1.6 \pm 0.8$ & $93.89 \pm 2.92$ & $* 9.08 \pm 2.82$ \\
\hline 3 & $* * 1290 \pm 20.41$ & $* * * 5.1 \pm 0.4$ & $\begin{array}{c}* 90.19 \\
\pm 1.15\end{array}$ & $\begin{array}{c}* * * 18.6 \\
\pm 1.29\end{array}$ \\
\hline 5 & $* * * 1225 \pm 30.1$ & $* * * 7.7 \pm 0.6$ & $* * * 78.41 \pm 1.1$ & $* * * 32.9 \pm 0.54$ \\
\hline 7 & $* * * 1135 \pm 14.29$ & $* * * 11.2 \pm 0.8$ & $* * * 55.5 \pm 3.4$ & $* * * 56.0 \pm 2.33$ \\
\hline 9 & $* * * 0 \pm 0.0$ & $0 \pm 0.0$ & $* * * 0 \pm 0.0$ & $0 \pm 0.0$ \\
\hline
\end{tabular}

On the other hand, the oviposition deterrent index (O.D.I) and percentages of sterility were positively correlated with the concentrations for instance; (O.D.I) was 1.6, 5.1, 7.7 and $11.2 \%$ at the concentrations of $1,3,5$ and $7 \mathrm{ppm}$, respectively. Also, the percentage of sterility was 9.08, 18.6, 32.87 and $56.03 \%$ at the previous concentrations.
Toxicological activities of flufenoxuron against $2^{\text {nd }}$ and $4^{\text {th }}$ larval instars of $S$. littoralis are summarized in Table (5). The corresponding concentration $\mathrm{LC}_{25}, \mathrm{LC}_{50}$ and $\mathrm{LC}_{90}$ were $0.1, \quad 0.2$ and $1.3 \mathrm{ppm}$, respectively for $2^{\text {nd }}$ instar. The corresponding concentrations $\mathrm{LC}_{25}$, $\mathrm{LC}_{50}$ and $\mathrm{LC}_{90}$ were $2.1,3.6$ and 9.8 ppm, respectively for $4^{\text {th }}$ instar. 
Table (5): Toxicity data of flufenoxuron against $2^{\text {nd }}$ and $4^{\text {th }}$ larval instars of S. littoralis.

\begin{tabular}{|c|c|c|c|c|}
\hline \multirow{2}{*}{ Conc. (ppm) } & \multicolumn{3}{|c|}{ Toxicity of flufenoxuron } & \multirow{2}{*}{ Slop function } \\
\cline { 2 - 4 } & $\mathbf{L C}_{\mathbf{2 5}}$ & $\mathbf{L C}_{\mathbf{5 0}}$ & $\mathbf{L C}_{\mathbf{9 0}}$ & 1.3 \\
\hline $\mathbf{2}^{\text {nd }}$ & 0.1 & 0.2 & 9.8 & 3 \\
\hline $\mathbf{4}^{\text {th }}$ & 2.1 & 3.6 & 9.8 & 3 \\
\hline
\end{tabular}

\section{Morphogenic abnormalities:}

The morphogenic abnormalities of larvae, pupae and adults which emerged from $2^{\text {nd }}$ and $4^{\text {th }}$ larval instars treated with the tested IGR could be grouped into five categories (malformed $2^{\text {nd }}$ larval instar, malformed $4^{\text {th }}$ larval instar, larval-pupal intermediates, malformed pupae and malformed adults). As compared with normal $2^{\text {nd }} \& 4^{\text {th }}$ larval instars, treatments with the different concentrations of the tested CSI were shown the presence of different degrees of abnormalities in larval stages.. As compared with normal $2^{\text {nd }}$ larval instar. and with normal $4^{\text {th }}$ larval instar, Treatments of $S$. littoralis larvae in both instars $2^{\text {nd }}$ and $4^{\text {th }}$ with the tested IR produced pupae with different degrees of morphogenic abnormalities such as pupa with C- shaped, pupae with a ring of larval cuticle around the abdomen and pupae with enlarged and shortened body . Some emerged adults have various degrees of morphogenic abnormalities.
Adults were unable to emerge from their pupal skins (failure adults' emergence), adults were completely free but possessed crumpled and incomplete formation of wings

\section{Enzymatic activities:-}

Enzymes were measured in treated and control groups of $2^{\text {nd }}$ and $4^{\text {th }}$ larval instars at $6,12,24$ and 48 hrs post treatment with flufenoxuron in order to determine the changes in these enzymes activity through flufenoxuron mode of action. The data recorded in Tables $(6,7,8)$ \& Figures (1-6) indicated that all the treatments with sub-lethal concentations $\left(\mathrm{LC}_{25}, \mathrm{LC}_{50}\right.$ and $\left.\mathrm{LC}_{90}\right)$ on $2^{\text {nd }}$ and $4^{\text {th }}$ larval instars at different time intervals have a positive effect on the activities of tested enzymes (acid phosphatase and $\alpha-\& \beta$ - esterases). The data declared that the activities were decreased with the increase of time and also with the increase in concentrations.

Table 6: Acid phosphatase activity of $2^{\text {nd }}$ and $4^{\text {th }}$ larval instars treated with sub-lethal concentrations of

\begin{tabular}{|c|c|c|c|}
\hline Larval & Dose & $\begin{array}{c}\text { Acid phosphatase activity } \\
(\mu \mathrm{g} \text { phenol released/b.wt./min) Mean } \pm \mathrm{SE}\end{array}$ & \\
\hline
\end{tabular}

Cascade at different time intervals. 


\begin{tabular}{|c|c|c|c|c|c|}
\hline stage & (ppm) & $\begin{array}{c}\text { Hours } \\
\text { post- treatment }\end{array}$ & Control & Treated & $\begin{array}{c}\text { Activity } \\
(\%)\end{array}$ \\
\hline \multirow{12}{*}{$\begin{array}{c}2^{\text {nd }} \text { larval } \\
\text { instar }\end{array}$} & \multirow{4}{*}{$\begin{array}{l}\mathbf{L} \mathbf{C}_{25} \\
(0.1)\end{array}$} & 6 & $2.0 \pm 0.01$ & $1.34 \pm 0.03^{* *}$ & -33 \\
\hline & & 12 & $9.445 \pm 0.2$ & $5.23 \pm 0.1^{* *}$ & -44.63 \\
\hline & & 24 & $11.86 \pm 0.24$ & $6.23 \pm 0.31^{* *}$ & -47.47 \\
\hline & & 48 & $13.47 \pm 0.41$ & $5.93 \pm 0.34^{* *}$ & -55.98 \\
\hline & \multirow{4}{*}{$\begin{array}{l}\mathbf{L C}_{50} \\
(0.2)\end{array}$} & 6 & $2.0 \pm 0.01$ & $1.0 \pm 0.1^{* *}$ & -50 \\
\hline & & 12 & $9.445 \pm 0.2$ & $3.68 \pm 0.32^{* *}$ & -61.04 \\
\hline & & 24 & $11.86 \pm 0.24$ & $4.98 \pm 0.31^{* *}$ & -58.01 \\
\hline & & 48 & $13.47 \pm 0.41$ & $4.93 \pm 0.34^{* *}$ & -63.4 \\
\hline & \multirow{4}{*}{$\begin{array}{l}\mathbf{L C}_{\mathbf{9 0}} \\
(1.3)\end{array}$} & 6 & $2.0 \pm 0.01$ & $0.8 \pm 0.001^{* *}$ & -60 \\
\hline & & 12 & $9.445 \pm 0.2$ & $2.96 \pm 0.21^{* *}$ & -68.66 \\
\hline & & 24 & $11.86 \pm 0.24$ & $3.89 \pm 0.34^{* *}$ & -67.2 \\
\hline & & 48 & $13.47 \pm 0.41$ & $3.98 \pm 0.55^{* *}$ & -70.45 \\
\hline \multirow{12}{*}{$\begin{array}{c}4^{\text {th }} \text { larval } \\
\text { instar }\end{array}$} & \multirow{4}{*}{$\begin{array}{l}\mathbf{L C}_{25} \\
(2.1)\end{array}$} & 6 & $5.979 \pm 0.05$ & $5.39 \pm 0.1^{* * *}$ & -9.85 \\
\hline & & 12 & $22.89 \pm 0.38$ & $18.63 \pm 0.47^{* *}$ & -18.61 \\
\hline & & 24 & $23.73 \pm 0.42$ & $16.68 \pm 0.58^{* * *}$ & -29.71 \\
\hline & & 48 & $26.94 \pm 0.53$ & $18.53 \pm 0.62^{* *}$ & -31.22 \\
\hline & \multirow{4}{*}{$\begin{array}{l}\mathbf{L C}_{\mathbf{5 0}} \\
(3.6)\end{array}$} & 6 & $5.979 \pm 0.05$ & $4.16 \pm 0.38^{* *}$ & -30.42 \\
\hline & & 12 & $22.89 \pm 0.38$ & $16.93 \pm 0.21^{* * *}$ & -26.04 \\
\hline & & 24 & $23.73 \pm 0.42$ & $15.85 \pm 0.71^{* *}$ & -33.21 \\
\hline & & 48 & $26.94 \pm 0.53$ & $16.38 \pm 0.62^{* * *}$ & -38.085 \\
\hline & \multirow{4}{*}{$\begin{array}{l}\mathbf{L C}_{\mathbf{9 0}} \\
(9.8)\end{array}$} & 6 & $5.979 \pm 0.05$ & $3.12 \pm 0.11^{* *}$ & -47.82 \\
\hline & & 12 & $22.89 \pm 0.38$ & $14.71 \pm 0.33^{* *}$ & -35.74 \\
\hline & & 24 & $23.73 \pm 0.42$ & $12.92 \pm 0.51^{* *}$ & -45.55 \\
\hline & & 48 & $26.94 \pm 0.53$ & $13.24 \pm 0.42^{* * *}$ & -50.85 \\
\hline
\end{tabular}

* Significant at $\mathrm{P}=0.05 \quad * *$ High significant at $\mathrm{P}=0.01 \quad * * *$ Very high significant at $\mathrm{P}=0.001$

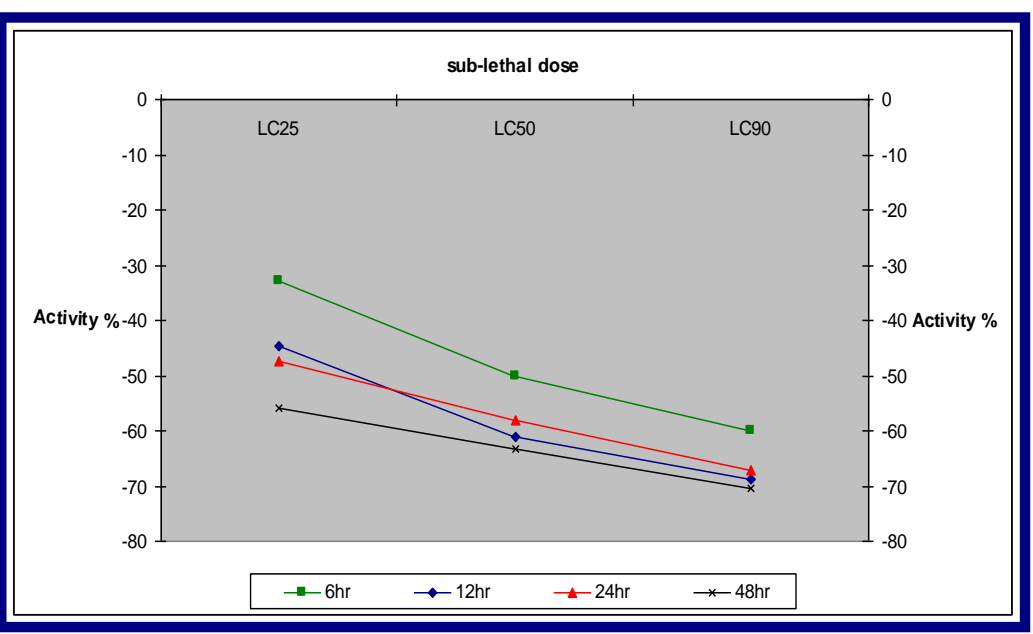

Fig. 1: Acid phosphatase activity of $2^{\text {nd }}$ larval instar treated with Sublethal doses of flufenoxuron at different time intervals.

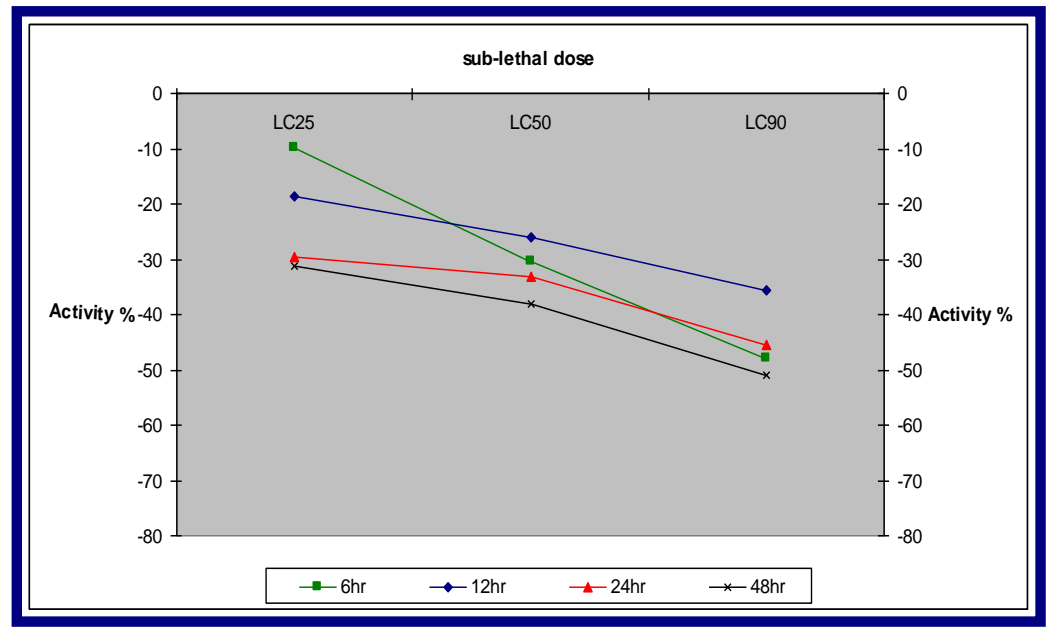

Fig. 2: Acid phosphatase activity of $4^{\text {th }}$ larval instar treated with Sub-lethal doses of flufenoxuron at different time intervals. 
Table (7): $\alpha$-Esterase activity of $2^{\text {nd }}$ and $4^{\text {th }}$ larval instars treated with sub-lethal concentrations of Cascade at different time intervals.

* Significant at $\mathrm{P}=0.05 * *$ High significant at $\mathrm{P}=0.01 * * *$ Very high significant at $\mathrm{P}=0.001$

\begin{tabular}{|c|c|c|c|c|c|}
\hline \multirow{2}{*}{$\begin{array}{c}\text { Larval } \\
\text { stage }\end{array}$} & \multirow{2}{*}{$\begin{array}{c}\text { Dose } \\
(\mathbf{p p m})\end{array}$} & \multicolumn{3}{|c|}{$\begin{array}{c}\alpha \text {-Esterase activity } \\
\text { ( } \mu \mathrm{g} \text { phenol released/b.wt./min) Mean } \pm \mathrm{SE}\end{array}$} & \multirow{2}{*}{$\begin{array}{c}\text { Activity } \\
(\%)\end{array}$} \\
\hline & & Hours post- treatment & Control & Treated & \\
\hline \multirow{12}{*}{$2^{\text {nd }}$ larval instar } & \multirow{4}{*}{$\begin{array}{r}\mathbf{L C}_{25} \\
(0.1)\end{array}$} & 6 & $464.835 \pm 2.64$ & $355.835 \pm 1.34$ & -23.45 \\
\hline & & 12 & $474.665 \pm 1.9$ & $347.142 \pm 2.1^{* *}$ & -26.86 \\
\hline & & 24 & $553.33 \pm 2.4$ & $397.69 \pm 2.4$ & -28.13 \\
\hline & & 48 & $673.335 \pm 3.4$ & $452.76 \pm 3.1^{* * *}$ & -32.76 \\
\hline & \multirow{4}{*}{$\begin{array}{l}\mathbf{L C}_{50} \\
(0.2)\end{array}$} & 6 & $464.835 \pm 2.64$ & $339.246 \pm 4.2^{* *}$ & -27.02 \\
\hline & & 12 & $474.665 \pm 1.9$ & $317.358 \pm 3.7^{* *}$ & -33.14 \\
\hline & & 24 & $553.33 \pm 2.4$ & $350.269 \pm 3.2^{* *}$ & -36.7 \\
\hline & & 48 & $673.335 \pm 3.4$ & $413.634 \pm 2.7^{\text {tim }}$ & -38.57 \\
\hline & \multirow{4}{*}{$\begin{array}{l}\mathbf{L} \mathbf{C}_{90} \\
(1.3)\end{array}$} & 6 & $464.835 \pm 2.64$ & $297.359 \pm 1.4^{* *}$ & -36.03 \\
\hline & & 12 & $474.665 \pm 1.9$ & $295.478 \pm 3.7^{* 7}$ & -37.75 \\
\hline & & 24 & $553.33 \pm 2.4$ & $306.943 \pm 1.3^{* * *}$ & -44.53 \\
\hline & & 48 & $673.335 \pm 3.4$ & $398.864 \pm 2.3^{*}$ & -40.8 \\
\hline \multirow{12}{*}{$4^{\text {th }}$ larval instar } & \multirow{4}{*}{$\begin{array}{r}\mathbf{L C} \mathbf{C}_{25} \\
(2.1)\end{array}$} & 6 & $749.57 \pm 3.6$ & $650.67 \pm 2.2^{2}$ & -13.19 \\
\hline & & 12 & $786.43 \pm 1.8$ & $638.78 \pm 4.1^{* *}$ & -18.77 \\
\hline & & 24 & $899.67 \pm 2.8$ & $717.33 \pm 2.4 *$ & -20.27 \\
\hline & & 48 & $976.83 \pm 2.4$ & $753.27 \pm 1.9^{* * *}$ & -22.89 \\
\hline & \multirow{4}{*}{$\begin{array}{l}\mathbf{L C} \mathbf{C}_{50} \\
(3.6)\end{array}$} & 6 & $749.57 \pm 3.6$ & $636.93 \pm 2.3^{* 2}$ & -15.03 \\
\hline & & 12 & $786.43 \pm 1.8$ & $597.96 \pm 4.6^{* *}$ & -23.96 \\
\hline & & 24 & $899.67 \pm 2.8$ & $651.89 \pm 1.7^{* * *}$ & -27.54 \\
\hline & & 48 & $976.83 \pm 2.4$ & $663.89 \pm 3.9^{* 7}$ & -32.04 \\
\hline & \multirow{4}{*}{$\begin{array}{l}\mathbf{L C}_{90} \\
(9.8)\end{array}$} & 6 & $749.57 \pm 3.6$ & $598.97 \pm 5.2^{* *}$ & -20.09 \\
\hline & & 12 & $786.43 \pm 1.8$ & $559.89 \pm 4.1^{* *}$ & -28.81 \\
\hline & & 24 & $899.67 \pm 2.8$ & $596.94 \pm 3.3^{* *}$ & -33.65 \\
\hline & & 48 & $976.83 \pm 2.4$ & $594.79 \pm 4.1^{* *}$ & -39.11 \\
\hline
\end{tabular}




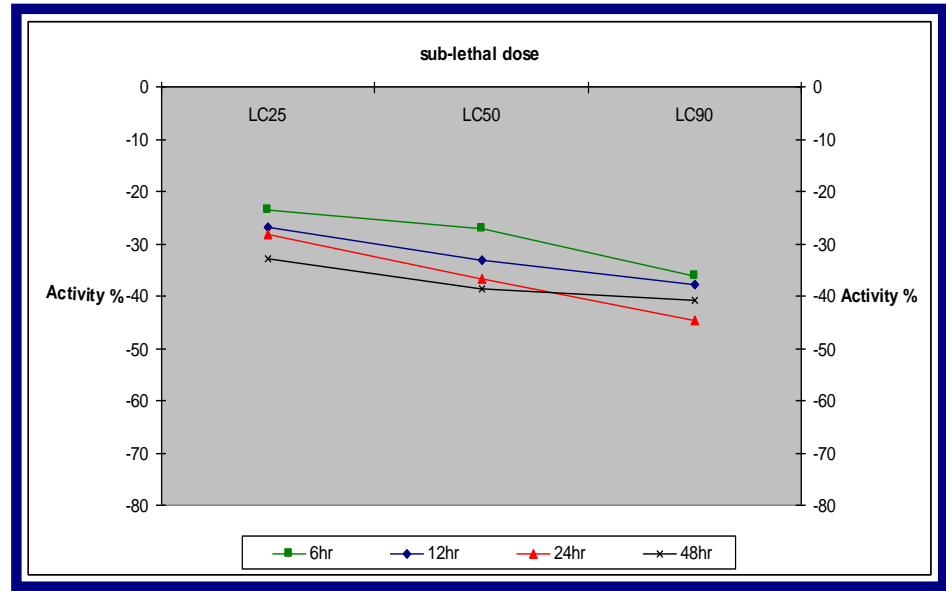

Fig. 3: $\alpha$-esterase activity of $2^{\text {nd }}$ larval instar treated with Sublethal doses of flufenoxuron at different time

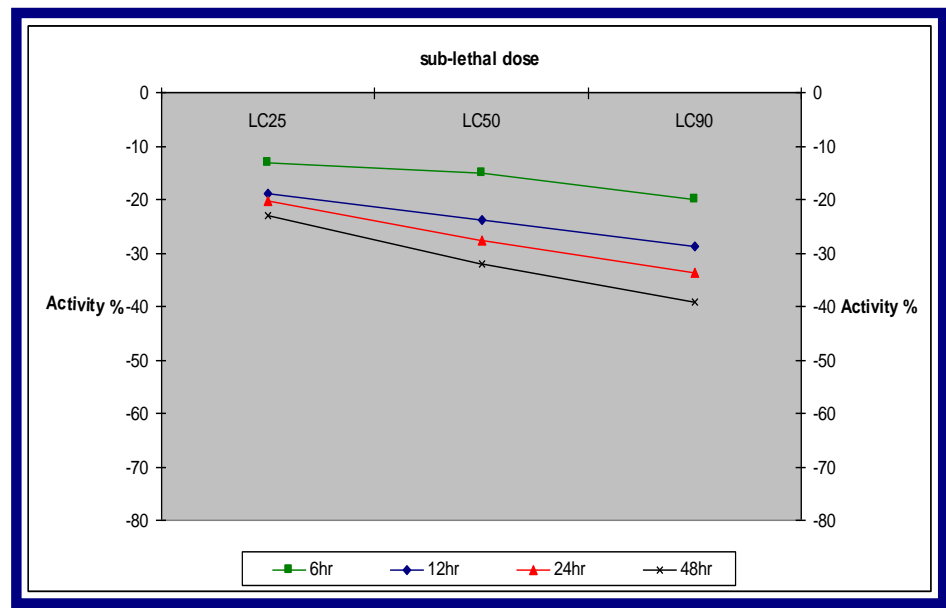

Fig. 4: $\alpha$-esterase activity of $4^{\text {th }}$ larval instar treated with Sublethal doses of flufenoxuron at different time intervals.

Table 8: $\beta$-Esterase activity of $2^{\text {nd }}$ and $4^{\text {th }}$ larval instars treated with sub-lethal concentrations of Cascade at different time intervals.

\begin{tabular}{|c|c|c|c|c|c|}
\hline \multirow{2}{*}{$\begin{array}{c}\text { Larval } \\
\text { stage }\end{array}$} & \multirow{2}{*}{$\begin{array}{c}\text { Dose } \\
(\mathbf{p p m})\end{array}$} & \multicolumn{3}{|c|}{$\begin{array}{c}\beta \text {-Esterase activity } \\
(\mu \mathrm{g} \text { phenol released/b.wt./min) Mean } \pm \mathrm{SE}\end{array}$} & \multirow[b]{2}{*}{ Activity (\%) } \\
\hline & & Hours post- treatment & Control & Treated & \\
\hline \multirow{12}{*}{$\begin{array}{l}2^{\text {nd }} \text { larval } \\
\text { instar }\end{array}$} & \multirow{4}{*}{$\begin{array}{r}\mathbf{L} \mathbf{C}_{25} \\
(0.1)\end{array}$} & 6 & $619.165 \pm 5.2$ & $543.5 \pm 3.7^{* * *}$ & -12.22 \\
\hline & & 12 & $842.665 \pm 4.4$ & $700.213 \pm 3.1^{* * *}$ & -16.9 \\
\hline & & 24 & $956.5 \pm 7.6$ & $724.44 \pm 5.8^{* * *}$ & -24.3 \\
\hline & & 48 & $1148.33 \pm 9.3$ & $815.32 \pm 4.3^{* * *}$ & -29.9 \\
\hline & \multirow{4}{*}{$\begin{array}{l}\mathbf{L} \mathbf{C}_{50} \\
(0.2)\end{array}$} & 6 & $619.165 \pm 5.2$ & $487.33 \pm 4.6^{* * *}$ & -21.3 \\
\hline & & 12 & $842.665 \pm 4.4$ & $620.42 \pm 6.4^{* *}$ & -26.4 \\
\hline & & 24 & $956.5 \pm 7.6$ & $680.4 \pm 6.1^{* *}$ & -28.9 \\
\hline & & 48 & $1148.33 \pm 9.3$ & $776.6 \pm 5.8^{* *}$ & -32.4 \\
\hline & \multirow{4}{*}{$\begin{array}{l}\mathbf{L} \mathbf{C}_{\mathbf{9 0}} \\
(1.3)\end{array}$} & 6 & $619.165 \pm 5.2$ & $409.22 \pm 2.2^{* * *}$ & -33.91 \\
\hline & & 12 & $842.665 \pm 4.4$ & $533.18 \pm 7.2^{* *}$ & -36.73 \\
\hline & & 24 & $956.5 \pm 7.6$ & $552.63 \pm 2.6^{* *}$ & -42.22 \\
\hline & & 48 & $1148.33 \pm 9.3$ & $613.72 \pm 5.8^{* * *}$ & -46.55 \\
\hline \multirow{12}{*}{$\begin{array}{c}4^{\text {th }} \text { larval } \\
\text { instar }\end{array}$} & \multirow{4}{*}{$\begin{array}{l}\mathbf{L} \mathbf{C}_{25} \\
(2.1)\end{array}$} & 6 & $1267.33 \pm 22.3$ & $1153 \pm 14.2^{*}$ & -9.02 \\
\hline & & 12 & $1734.28 \pm 32.1$ & $1542.3 \pm 20.05^{* *}$ & -11.07 \\
\hline & & 24 & $1913.42 \pm 17.4$ & $1596.82 \pm 15.32^{* *}$ & -16.55 \\
\hline & & 48 & $1125.48 \pm 24.3$ & $1073.6 \pm 13.5^{*}$ & -4.09 \\
\hline & \multirow{4}{*}{$\begin{array}{l}\mathbf{L} \mathbf{C}_{50} \\
(3.6)\end{array}$} & 6 & $1267.33 \pm 22.3$ & $1096.93 \pm 14.1^{*}$ & -13.44 \\
\hline & & 12 & $1734.28 \pm 32.1$ & $1406.75 \pm 18.01^{* *}$ & -18.9 \\
\hline & & 24 & $1913.42 \pm 17.4$ & $1485.74 \pm 18.4^{* *}$ & -22.35 \\
\hline & & 48 & $1125.48 \pm 24.3$ & $1002.8 \pm 16.2^{*}$ & -10.9 \\
\hline & \multirow{4}{*}{$\begin{array}{l}\mathbf{L C}_{\mathbf{9 0}} \\
(9.8)\end{array}$} & 6 & $1267.33 \pm 22.3$ & $984.34 \pm 12.7^{* *}$ & -22.33 \\
\hline & & 12 & $1734.28 \pm 32.1$ & $1300.82 \pm 21.4^{* *}$ & -25 \\
\hline & & 24 & $1913.42 \pm 17.4$ & $1347.33 \pm 18.4^{* * *}$ & -29.6 \\
\hline & & 48 & $1125.48 \pm 24.3$ & $987.53 \pm 12.7^{*}$ & -12.26 \\
\hline
\end{tabular}

\footnotetext{
* Significant at $\mathrm{P}=0.05 \quad * *$ High significant at $\mathrm{P}=0.01 * * *$ Very high significant at $\mathrm{P}=0.001$
} 


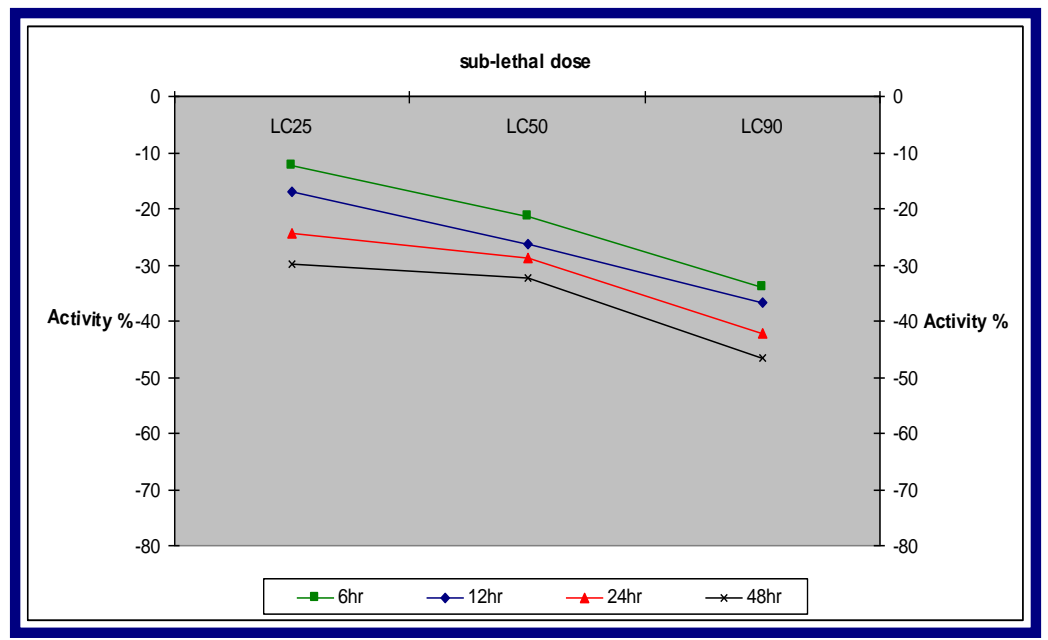

Fig. 5: $\beta$-esterase activity of $2^{\text {nd }}$ larval instar treated with Sub-lethal doses of flufenoxuron at different time intervals.

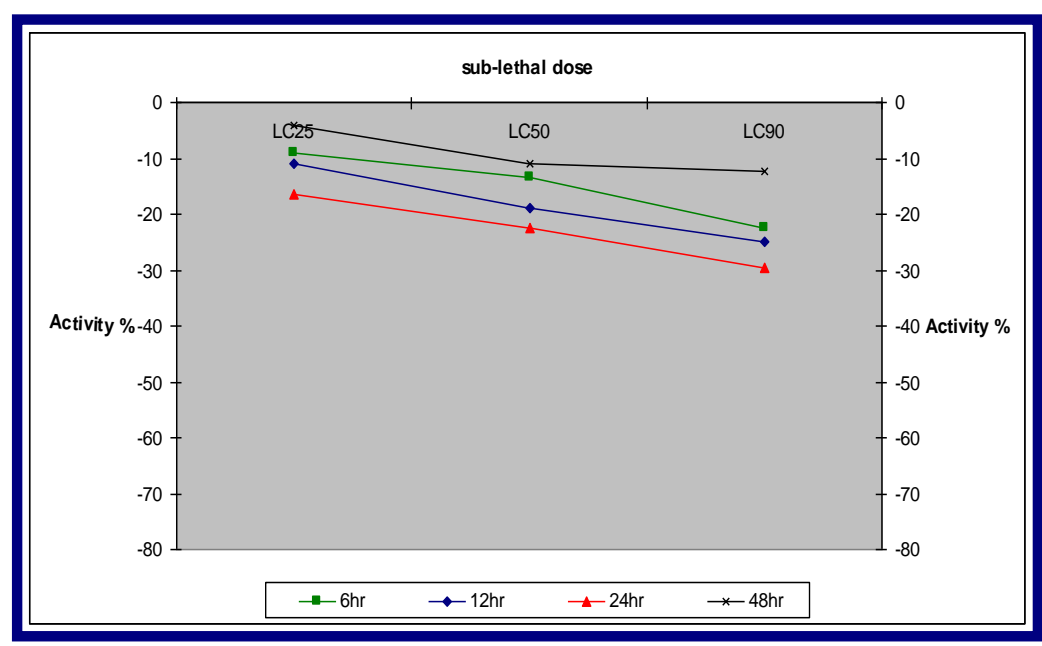

Fig. 6: $\beta$-esterase activity of $4^{\text {th }}$ larval instar treated with Sub-lethal doses of flufenoxuron at different time intervals.

\section{DISCUSSION}

In the present study the Chitin synthesis inhibitors, (flufenoxuron) caused appreciable toxic effect in larvae of S. littoralis. The response of larval mortalities caused by these CSI in the present investigation is similar to the results obtained by (Hussain, 1992; Smagghe et al., 1995; Whiting et al., 2000 and Saenz-de-Cabenzon et al., 2004 ).

Flufenoxuron is chitin synthesis inhibitor involved in insect growth and development during molting, due to its lipophilic properties it can interfere with the exoskeleton chitin by contact. Furthermore higher concentrations have antifeeding effect. Chitin synthesis inhibitors found to be effect on the vira-like chitinase gene which responsible for producing chitinolytic enzyme work in remodeling chitinous structures known as glycanohydrolase, catalyze the hydrolysis of $[\beta-(1-4)$ glycoside] bonds of chitin polymers and oligomers (Konodo et al., 2002), which involved in chitin degredation, as well as this compound effect also on the gene which responsible for production of glycolytic enzyme, triosephosphate isomerase, which involved in catalyzes the interconversion of 
dihydroxyacetone phosphate and Dglyceraldehyde-3-phosphate, the alimentary canal is lined with cuticle which formed from chitin, proteins, lipids and hydrocarbons, thus the alimentary canal (fore and hind gut) of the treated larvae is the first position to be affected with these compounds, as well as the mid gut (peritrophic membrane), chitinases seem to be involved in the formation, perforation and degredation of the midgut peritrophic matrix, which protect the gut epithelium from damaging factors (Filho et al., 2002).

Generally, the $2^{\text {nd }}$ larval instar was found to be more sensitive to the tested compound than $4^{\text {th }}$ instar. The obtained low values of slop function indicated the homogenous response of the treated larvae to different concentrations of the tested compounds. The above obtained results were in agreement with those obtained by (Badr, 2000; Culter et al., 2005 and Han et al. 2006).

The $4^{\text {th }}$ larval instar tolerance could be due to the changes in anatomy, physiology and size through which the compounds passes, or may be due to difference in liability to toxicant penetration (Busvine, 1971).

Pupal mortalities in this study were obvious and recorded after treatment of both $2^{\text {nd }}$ and $4^{\text {th }}$ larval instars with the used CSI, there were dose-dependent effect on pupation and pupal mortalities, these results are in harmony with the results obtained by (Whiting et al., 2000 ; Butter et al., 2003 ; Biddinger et al., 2006 and Salokhe et al., 2008 ).

Total inhibition of adult emergence in the biological studies were recorded for the treated larvae with the used CSI, it was obvious that the percents of inhibition were in positive relationship with the increase of concentrations, these results are in agreement with those obtained by (Butter et al., 2003; Biddinger et al., 2006 ; Salokhe et al., 2008 and Wang \&Tian 2009).

Reduction in fecundity in the present study was recorded for the resulted female moths treated as $2^{\text {nd }}$ and $4^{\text {th }}$ larval instars for the tested CSI, these obtained results are in agreement with other authors (Butter et al., 2003; Saenz-de-Cabenzon et al., 2004 ; Khebbeb et al., 2008 ; Wang and Tian 2009). The reduction in total number of eggs per female in this study could be due to interference of the tested CSI with oogenesis; they induces decrease in the concentration of yolk proteins, carbohydrates, lipids and inhibition in both DNA and RNA synthesis in the ovaries of females treated as larval instars, moreover they caused vacuolation of nurse cells and oocytes of the ovaries (Shaurub et al., 1998).

Also reduction in fecundity may be due to the reduction in longevity and the number of oocytes per ovary and the reduction in oviposition period ( Soltani and Mazouni, 1992). In addition to the above factors the maturation of an insect egg depend on the materials that are taken up from the surrounding haemolemph and materials synthesized by the ovary in suit, these materials includes protein, lipids and carbohydrates all of which required for embryonic structure (Soltani and Mazouni, 1992 and Shaurub et al ., 1999).

Reduction in the percentage of egg- hatch obtained in the present study could be due to sterilization of both eggs and sperms or may be due to inability of the sperms to be transferred to females during copulation (Ismail, 1980).

Ovicidal activity of the tested CSI in the present study could be due 
to the disturbance in cuticle formation of the embryo, (Sallam 1999), developed embryos were enabled to perforate the surrounding vitelline membrane, it could be due to a weakened chitinous mouth parts that was insufficiently rigid to effect hatching. Inhibitory effect of the tested CSI on the acid phosphates in the present study was observed, and these obtained results are in harmony with these results investigated by Mostafa 1993. Acid phosphatase has been shown to be associated with insect development especially in relation to nutrition and egg maturation (Ali 2008).

The present study showed that the activities of $\alpha$-esterase and $\beta$ esterase were reduced significantly in treated larvae as compared with control, the results showed that the reduction in activity was positively correlated to increase in dose and time post treatments these results are in agreement with (Abdel-Hafez et al. 1993 and Ali 2008).

Inhibition of non specific esterase's enzymes could be suggested the reduction in fecundity and fertility, and they could be playing a role in the metamorphic inhibition.

\section{REFERENCES}

Abdel-Hafez, M. M.; Mohanna, A.; Afifi, M. A. and Eid. A. H. (1993): Effect of IGR/insecticide mixtures on esterases activity of Spodoptera littoralis. J. Product. And Dev., 1 (2): 153-164.

Abo-Arab, R.B. and Salem, A. A. (2005): Efficiency of some plant extracts compared to pirimiphos-methyl as insecticidal and ovicidal agents. Alex. J. Agric. Res., 50 (2): 53-60.
Ali, M.M. (2008): Biochemical and physiological studies on the cotton leafworm Spodoptera littoralis. M.Sc. Thesis, Fac. Sci., Benha Univ., Egypt.

Badr, N.A. (2000): Efficacy of some natural products and insect growth regulators, Consult against the cotton leafworm, Spodoptera littoralis Bosid. Egypt. J. Appl. Sci., 15 (9): 316-327.

Biddinger, D.; Hull, L.; Huang, H.; Mcpheron, B. and Loyer, M. (2006): Sublethal effects of chronic exposure to Tebufenozide on the development, survival and reproduction of the tufted apple bud moth. J. Econ. Entomol. 99(3): 834-842.

Busvine, J.R. (1971): A critical review of techniques for testing insecticides. Commonwealth Agric. Bureau, England, 345pp.

Butter, N.S.; Gurmeet S. and Dhawanm A.K. (2003): Laboratory evaluation of the insect growth regulator Lufenuron against Helicoverpa armigera on Cotton. Phytoparasitica, 31(2): 56-60.

Culter, G.C.; Scott-Dupree, C.D.; Tolman, J.H. and Harris, C.R. (2005): Acute and sublethal toxicity of Novaluron, a novel chitin synthesis inhibitor, to Leptinotarsa decemlineata (Coleo.: Chrysomelidae). Pest Manag. Sci., 61 (11): 1060- 1068.

DeLoach, J.R.; Meola, S.M.; Mayer, R.T. and Thompson, J.M. (1981): Inhibition of DNA synthesis by diflubenzuron in pupae of the stable fly 
Stomoxys calcitrans (L.) Pest. Biochem. Physiol., 15:172.

El-Barky N.M; Amer A.E. and Mervet A.K. (2009): Ovicidal activity and biological effects of radiation and Hexaflumeron against eggs of pink bollworm Pectinophora gossypiella (Saunders) ( Lepidoptera : Gelechiidae). Egypt. J. biolog. Sci., 2(1): 23-36.

Filho, B.P.; Lemos, F.J.; Secundino,

N. F.; Pascoa, V.; Pereira, S.T. and Pimenta, P.F. (2002): Presence of chitinase and betaNacetylglucosaminidase in the Aedes aegypti: a chitinolytic system involving peritrophic matrix formation and degradation. Insect Biochem. Mol. Biol. 32, 1723-1729.

Gijswijt, M.J.; Deul, D.H. and DeJong, B.J. (1979): Inhibition of chitin synthesis by benzoylphenylurea insecticides, III. Similarity in action in Pieres brassicae (L.) with polyxin D. Pestic. Biochem. Physiol., 12:84-94.

Hammock, C.D. and Quisted, G.B. (1981): Metabolism and mode of action of juvenile hormone, juvenoids and other insect growth regulators. In "Progress in pesticide Biochemistry" (Hutson, D.H. and Roberts, T.R. eds.), Vol. 1, pp. 1-85, John Wiley \& Sons Ltd.

Han, M.; Kim, S. and Ahn, Y. (2006): Insecticidal and antifeedant activities of medicinal plant extracts against Attagenus unicolor japonicus. J. Stored Prod. Resh., 42(1): 15-22.

Hosny, M.M.; Topper, C.P.; Moawad, G.G. and El-Saadany, G.B.
(1986): Economic damage thresholds of Spodoptera littoralis Bosid (Lepidoptera: Noctuidae) on cotton in Egypt. Crop. Prot. 5: 100104.

Hussain, N.H. (1992): Biochemical effect of Flufenoxuron on pink bollworm larvae, $\mathrm{P}$. gossypiella (Saund). Al Azhar J. Agric. Res., 16: 193-204.

Ismail, I.E. (1980): Physiological studies on the effect of juvenile hormone analogues upon the cotton leafworm, Spodoptera littoralis. Ph. D. Thesis, Cairo Univ., Egypt.

Khebbeb, M.E.H.; Gaouaoui, R. and Bendjeddou, F. (2008): Tebufenozide effects on the reproductive potentials of the Mediterranean flour moth, Ephestia kuehniella. African Journal of Biotechnology. 7(8): 1166-1170.

Kondo, K., Matsumoto, M., Kojo, A. and Mauda, R. (2002): Purification and characterization of chitinase from pupae Pieris rapae crucivora (Boiduval). J. Chem. Eng. Jap. 35, 241-246.

Magd El-Din and El-Gengaihi, S.E. (2000): Joint action of some botanical extracts against the Egyptian cotton leafworm Spodoptera littoralis Bosid (Lepidoptera: Noctuidae). Egypt. J. Biol. P. Cont. 10 (1): 51-56.

Mostafa, S. A. (1993): Biochemical effect of some chemical compounds on $S$. littoralis (Boisd.). Ph. D. Thesis. Fac. Agric. Al-Azhar. Univ. Egypt. 
Nasr, F. N. (1999): New isolated Bacillus spp. against the cotton leafworm, Spodoptera littoralis (Boisd.) (Lepidoptera: Noctuidae). Egypt. J. Agric. Res. 77 (4):1573-1583.

Pineda, S.; Schneider, MI.; Smagghe, G.; Martínez, AM.; Del Estal, P.; Viñuela, E.; Valle, J. and Budia, F. (2007): Lethal and sublethal effects of methoxyfenozide and spinosad on Spodoptera littoralis (Lepidoptera: Noctuidae). J. Econ. Entomol.; 100(3):773-780.

Powell, M. E. A. and M. J. H. Smith (1954): The determination of serum acid and alkaline phosphatase activity with 4aminoantipyrine. J. Clin. Pathol., 7: 245-248.

Saenz-de-Cabenzon Irigaray, F.J.; Marco, V. ; Zalom, F.G. and Perez Moreno, I. (2004): Effect of lufenuron on Lobesia botrana. Pest. Manag. Sci., 61(11): 1133$1137 .$.

Sallam, M.H. (1999): Effect of Diflubenzuron on embryonic development of the acridid, Heteracris littoralis. J. Egypt. Ger. Soc. Zool., 30(E):17-26.

Salokhe, S.G.; Pal, J.K. and Mukherjee S.N. (2008): Effect of sublethal concentrations of flufenoxuron on development, growth and reproductive performance of Tribolium castaneum. Journal of Invertebrate reproduction\&development. 43(2): 141-150.

Schmutterer, H. (1985): Which insect bests can be controlled by application of neem seed kernel extracts under field conditions? Z.ang. Ent., 100: 458-475.

Shaurub, E.H.; Ahmed, Z.A. and Samira, E.M. (1998): Impacts of Pyriproxyfen and extracts of Schinus terebinthifolius, on development, reproduction and reproductive organs in Spodoptera littoralis. J. Egypt. Ger. Soc. Zool.,27(E): 57-82

Soltani, N. and Mazouni, A. (1992): Diflubenzuron and oogenesis in the codling moth, Cydia pomonella. Pesti. Sci., 34: 257-261.

Smaggha, G.; Audenaert, L. and Degheele, D. (1995): Tebufenozide is toxicity correlated with pharmacokinetics and metabolism of different strains of the Egyptian cotton leafworm. MededelingenFaculteiesLandbouwkundige-enToegepaste-BiologischeWetenschappen-Univ.-Gent., 60(3b): 1015-1016.

Van Asperen, R. (1962): A study of house flies esterase by means of sensitive colourimetric method. J. Insect. Physiol., 8: 401-416.

Verloop, A. and Ferrel, C.D. (1977): Benzoylureas - a new group of larvicides interferring with chitin deposition. In "Pesticide chemistry in the 20th Century" (Plummer, J.R., ed.). Acs symposium series 37, pp: 237-270, Whashington, D.C., Amer. Chem.Soc.

Wang J. and Tian D. (2009): Sublethal effects of Methoxyfenozide on Spodoptera litura. Cotton Science, 21(3): 212-217. 
Whiting D.C.; Jamieson L.E. and Connolly P.G. (2000): Prepostvittana (Lepid. Tortricidae). J. J. Econ. and postharvest effect of Entomol. 93 (3): 673-679. Lufenuron on Epiphyas

\section{ARABIC SUMMARY}

تأثير مثبط تكوين الكيتين (فلوفينوكسيرون) على بعض النواحى البيولوجية والكيموحيوية لدودة ورق القطن الكبرى النو

رضا فضيل على بكر 1 ـ نهاد محمد البرقىـ محمد2 - منى فوزي عبد العزيز2 ـ ـ محد حسين عواد 3 ـ

هشام محمد السيا عبدالحئيم2 2

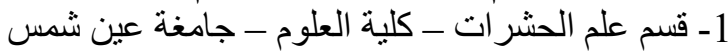

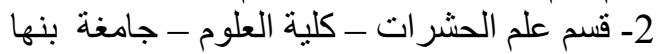

3- قسم علم الحيو ان - كلية العلوم - جامعة بنها

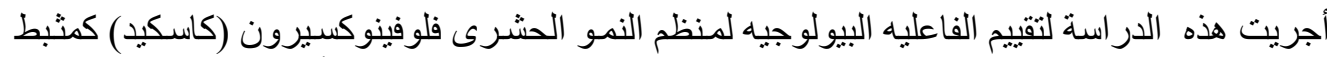

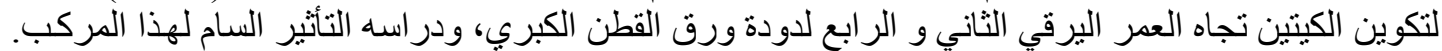

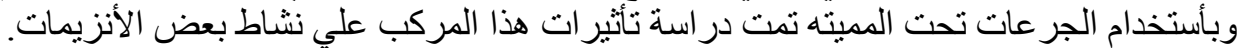

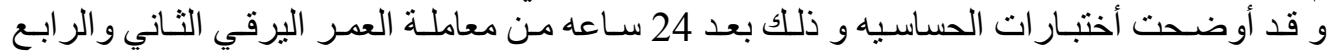

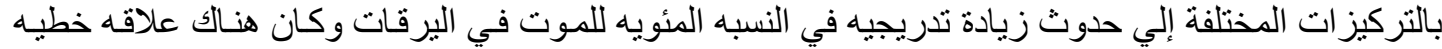

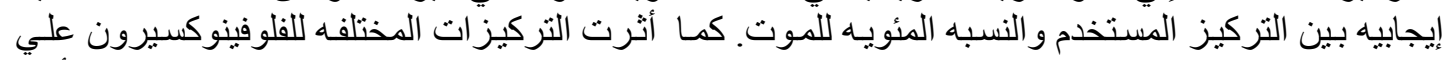

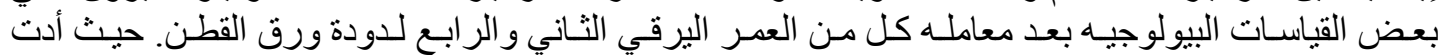

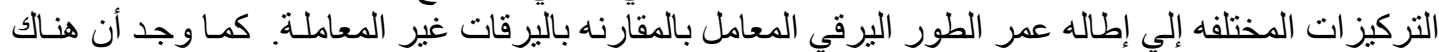

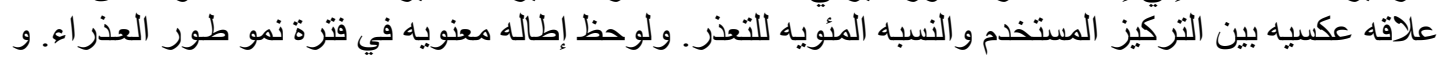

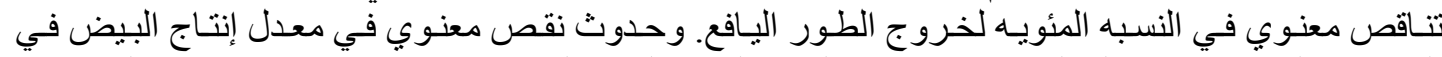

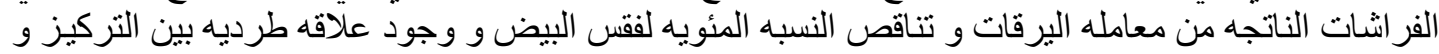

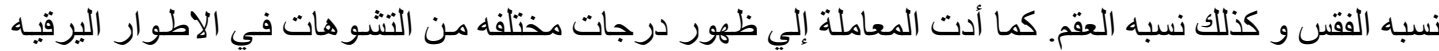

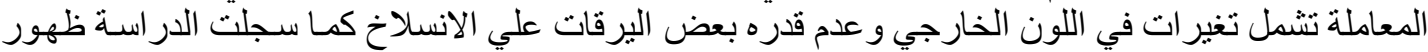

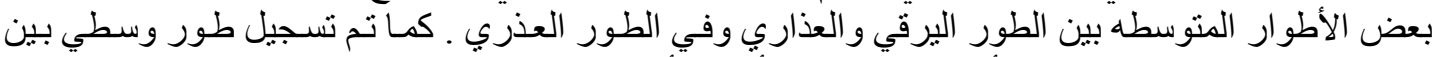

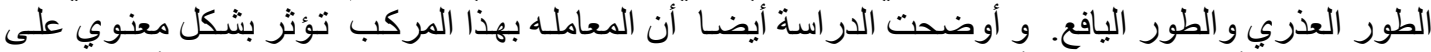

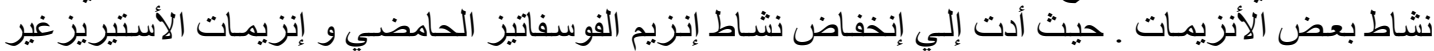
المتخصصه (ألفاو بيتا) في اليرقات المعاملة بالمقارنه باليرقات إلئن غبر المعاملة. 